\title{
Computer vision based analysis of potato chips - A tool for rapid detection of acrylamide level
}

\author{
Vural Gökmen ${ }^{1}$, Hamide Z. Senyuva ${ }^{2}$, Berkan Dülek $^{3}$ and Enis Çetin ${ }^{3}$ \\ ${ }^{1}$ Department of Food Engineering, Hacettepe University, Beytepe, Ankara, Turkey \\ ${ }^{2}$ Ankara Test and Analysis Laboratory, Scientific and Technical Research Council of Turkey, Ankara, \\ Turkey \\ ${ }^{3}$ Department of Electrical and Electronics Engineering, Bilkent University, Bilkent, Ankara, Turkey
}

In this study, analysis of digital color images of fried potato chips were combined with parallel LCMS based analysis of acrylamide in order to develop a rapid tool for the estimation of acrylamide during processing. Pixels of the fried potato image were classified into three sets based on their Euclidian distances to the representative mean values of typical bright yellow, yellowish brown, and dark brown regions using a semiautomatic segmentation algorithm. The featuring parameter extracted from the segmented image was NA2 value which was defined as the number of pixels in Set-2 divided by the total number of pixels of the entire fried potato image. Using training images of potato chips, it was shown that there was a strong linear correlation $(r=0.989)$ between acrylamide level and NA2 value. Images of a number of test samples were analyzed to predict their acrylamide level by means of this correlation data. The results confirmed that computer vision system described here provided explicit and meaningful description from the viewpoint of inspection and evaluation purpose for potato chips. Assuming a provisional threshold limit of $1000 \mathrm{ng} / \mathrm{g}$ for acrylamide, test samples could be successfully inspected with only one failure out of 60 potato chips.

Keywords: Acrylamide / Computer vision / Image analysis / Potato chips

Received: December 9, 2005; revised: January 25, 2006; accepted: February 18, 2006

\section{Introduction}

High concentrations of acrylamide found in common fried and baked foods attained considerable public concern since it has been classified as a probable human carcinogen $[1,2]$. To date, many analytical methods have been published for the analysis of acrylamide in thermally processed foods [3-10]. Although these methods may perform well for quality control purposes in a food analysis laboratory, they are laborious, costly, and cannot be adopted easily for process control purposes by the food industry. To satisfy the increased awareness, and greater expectation of consumers, as well as demands by the regulatory authorities, it is necessary to improve the evaluation quality of food products. Being an objective, rapid, and noncontact tool, computer vision may be a powerful technique for inspection and evaluation purposes by a rapid prediction of acrylamide level in food products.

Computer vision is the construction of explicit and meaningful descriptions of physical objects from images [11].

Correspondence: Dr. Vural Gökmen, Department of Food Engineering, Hacettepe University, Beytepe 06532, Ankara, Turkey

E-mail: vgokmen@hacettepe.edu.tr

Fax: $+90-312-2892123$
Image analysis is the core of computer vision with numerous algorithms and methods available to achieve the required classification and measurements [12]. From the viewpoint of acrylamide, surface color is an important food attribute that can be used to predict the acrylamide level.

It is the fact that both brown colored products and acrylamide are formed during the Maillard reaction at high temperatures [13-17]. Previous findings profoundly suggest that surface color may be correlated with acrylamide in thermally processed foods [18-20]. Earlier attempts were directed to measure color in Lab color space units which is an international standard for color measurements, adopted by the Commission Internationale d'Eclairage (CIE) in 1976. Although CIE redness parameter was shown to be correlated with the level of acrylamide to a certain extent, it may not be a reliable predictor of acrylamide concentration in potato chips due to nonhomogenous surface color.

This paper describes a computer vision based image analysis algorithm for the prediction of acrylamide level in potato chips. Training images were used to extract a meaningful parameter to be correlated with acrylamide level in potato chips. Images were segmented into three sets reflecting bright yellow, yellowish brown, and dark brown regions based on the Euclidian distance of each pixel to the refer- 
ence means defined during the training process. Test samples $(n=60)$ were analyzed using the semiautomatic segmentation algorithm to predict their acrylamide level.

\section{Materials and methods}

\subsection{Chemicals and consumables}

Acrylamide (99+\%) and ${ }^{13} \mathrm{C}_{3}$-labeled acrylamide (99\% isotopic purity) were obtained from Sigma (Diesenhofen, Germany) and Cambridge Isotope Laboratories (Andover, MA, USA), respectively. Methanol, potassium hexacyanoferrate, zinc sulfate, formic acid (98\%), and acetic acid (glacial) were of analytical grade and obtained from Merck (Darmstadt, Germany). Ultrapure water was used throughout the experiments (MilliQ system, Millipore, Bedford, MA, USA). Oasis HLB (1 mL, $30 \mathrm{mg}$ ) SPE cartridges were supplied by Waters (Milford, MA, USA). Glass vials with septum screw caps were supplied by Agilent Technologies (Wilmington, DE, USA). The analytical column (Inertsil ODS-3, $250 \mathrm{~mm} \times 4.6 \mathrm{~mm}, 5 \mu \mathrm{m})$ was supplied by HiChrom (Berkshire, UK).

Stock solution of acrylamide $(1 \mathrm{mg} / \mathrm{mL})$ and ${ }^{13} \mathrm{C}_{3}$-labeled acrylamide $(0.1 \mathrm{mg} / \mathrm{mL})$ was prepared by dissolving in distilled water. Working standards were prepared by diluting the stock solution of acrylamide to concentrations of 0.1 , $0.2,0.3,0.5,1.0$, and $2.0 \mu \mathrm{g} / \mathrm{mL}$ with $0.01 \mathrm{mM}$ acetic acid. Stock solutions and working standards were kept at $4{ }^{\circ} \mathrm{C}$ for a month. Carrez I solution was prepared by dissolving $15 \mathrm{~g}$ of potassium hexacyanoferrate in $100 \mathrm{~mL}$ of water, and Carrez II solution by dissolving $30 \mathrm{~g}$ of zinc sulfate in $100 \mathrm{~mL}$ of water.

\subsection{Preparation of potato chips for training}

Potato tubers were washed, peeled, and cut into $2 \mathrm{~mm}$ thickness slices using a slicer. Slices were fried in oil bath (Precisterm, J.P. Selecta, Spain) at different time-temperature combinations to obtain training samples with surface colors ranging from bright yellow to dark brown. Frying temperature was adjusted to $150(3,5,8,10$, and $15 \mathrm{~min}), 170(3,5$, and $8 \mathrm{~min})$, and $190^{\circ} \mathrm{C}$ ( 1 and $3 \mathrm{~min}$ ) to prepare ten pieces of training samples. Fried chips were drained over a wire screen for $5 \mathrm{~min}$ to remove excess oil and then the samples were photographed.

\subsection{Image acquisition and processing}

Images were captured with a color digital camera (HewlettPackard model R507) at its maximum resolution $(2048 \times 1536$ pixels $)$. The camera was located vertically at a distance of $25 \mathrm{~cm}$ from the sample. Captured images were stored in a PC in jpeg format without compression.

In fried potato chip images, there were three regions which typically have bright yellow, yellowish brown, and dark brown colors. After the representative mean red, green, and blue values of the pixels of these three regions were determined, pixels of the fried potato images were classified into three sets based on their Euclidian distances to the representative mean values. This process is also called vector quantization [21,22]. A Matlab code called VectorQuantize was implemented to process potato chip images pixel by pixel (Appendix). Based on this, the entries of Set-I, Set-II, and Set-III pixels which correspond to bright yellow, yellowish brown, and dark brown regions, respectively, were determined by means of a semiautomatic segmentation algorithm.

\subsection{Measurement of acrylamide}

\subsubsection{Sample preparation}

A sample preparation procedure previously described by us elsewhere was used [23]. Finely ground potato chips or French fries were weighed $(1 \mathrm{~g})$ into a $10 \mathrm{~mL}$ glass centrifuge tube with cap. ${ }^{13} \mathrm{C}_{3}$-labeled acrylamide $(1000 \mathrm{ng} / \mathrm{g}$ ) was introduced at this stage to confirm the results. The sample was suspended in $5 \mathrm{~mL}$ of methanol and extracted for $2 \mathrm{~min}$ in a homogenizer. The extract was centrifuged at $5000 \mathrm{rpm}$ for $10 \mathrm{~min}$. The clear supernatant was transferred into a centrifuge tube and treated with Carrez I and II solutions $(25 \mu \mathrm{L}$ each) to precipitate the coextractives. Following centrifugation at $5000 \mathrm{rpm}$ for $5 \mathrm{~min}, 1.0 \mathrm{~mL}$ of clear supernatant ( $0.2 \mathrm{~g}$ sample) was quantitatively transferred into a conical bottom glass test tube placed in a water bath at $40^{\circ} \mathrm{C}$ and evaporated to a final volume of $c a .50 \mu \mathrm{L}$ under nitrogen at $3 \mathrm{psig}$. After completing the volume to $1.0 \mathrm{~mL}$ with water, it was vortexed for $1 \mathrm{~min}$. For the SPE cleanup, Oasis HLB cartridge was preconditioned consequently with $1 \mathrm{~mL}$ of methanol and $1 \mathrm{~mL}$ of water at the rate of two drops per second using a syringe. Then, $1 \mathrm{~mL}$ of the extract was passed through the cartridge at a rate of one drop per second using a syringe. The first ten drops of the effluent were discarded to prevent any dilution of sample by replacing water held in the sorbent void fraction with the sample effluent. The forthcoming drops were collected and filtered through a $0.45 \mu \mathrm{m}$ syringe filter. Twenty microliters of the final test solution was injected onto LC column for quantification by LC-MS.

\subsubsection{LC-MS analysis}

LC-MS analyses were performed by an Agilent 1100 HPLC system consisting of a binary pump, an autosampler, and a temperature controlled column oven, coupled to an Agilent 
1100 MS detector equipped with atmospheric pressure chemical ionization interface. The analytical separation was performed using the isocratic mixture of $0.01 \mathrm{mM}$ acetic acid in $0.2 \%$ aqueous solution of formic acid at a flow rate of $0.6 \mathrm{~mL} / \mathrm{min}$ at $25^{\circ} \mathrm{C}$. The LC eluent was directed to the MS system after a delay time of $6.5 \mathrm{~min}$ using MSD software. Data acquisition was performed in SIM mode using the interface parameters: drying gas $\left(\mathrm{N}_{2}, 100\right.$ psig) flow of $4 \mathrm{~L} / \mathrm{min}$, nebulizer pressure of $60 \mathrm{psig}$, drying gas temperature of $325^{\circ} \mathrm{C}$, vaporizer temperature of $425^{\circ} \mathrm{C}$, capillary voltage of $4 \mathrm{kV}$, corona current of $4 \mu \mathrm{A}$, and fragmentor voltage of $55 \mathrm{eV}$. Ions monitored were $m / z 72$ and 55 for acrylamide and $\mathrm{m} / \mathrm{z} 75$ and 58 for ${ }^{13} \mathrm{C}_{3}$-labeled acrylamide for the quantification of acrylamide in the samples.

\section{Results and discussion}

Change of color in potato chips is a dynamic process in which certain color transitions occur as the frying proceeds. Digital image pixel values can be used to estimate acrylamide level of a potato chip after a useful feature is extracted from the digital image. After a frying process, three different kinds of image pixels which correspond to bright yellow, yellowish brown, and dark brown regions were observed in the digital images of training samples. It was experimentally observed that the yellowish brown colored potato chips had the highest level of acrylamide. In this paper, a relation between the acrylamide levels in a fried potato chip and its image has been established.

In this section, details of digital image processing and the Matlab program implemented are described. A typical image captured by a digital camera consists of an array of vectors called pixels. Each pixel $x[n, m]$ has red, green, and blue color values:

$x[n, m]=\left[\begin{array}{l}x_{r}(n, m) \\ x_{g}(n, m) \\ x_{b}(n, m)\end{array}\right]$

where $x_{r}(n, m), x_{g}(n, m)$, and $x_{b}(n, m)$ are values of the red, green, and blue components of the $(m, n)^{\text {th }}$ pixel $x[n, m]$, respectively. In digital images, $x_{r}, x_{g}$, and $x_{b}$ color components are represented in 8 bits, i.e., they are allowed to take integer values between 0 and 255 $\left(=2^{8}-1\right)$ [24].

The image saved in file_name is stored in matrix im_seq by converting integer values to doubles using the following instructions:

im_seq = imread('file_name');

im_seq $=$ im2double $($ im_seq $)$;

Using the training images of potato chips, the representative mean $x_{r}, x_{g}$, and $x_{b}$ color components of bright yellow, yel- lowish brown, and dark brown regions were determined. First, the image of interest stored in matrix im_seq was displayed in a window and the region of interest was selected by the following instructions:

$\operatorname{Imshow}($ im_seq);

Region_n = squeeze(imcrop);

After a successful training process, the mean representative values for the red, green, and blue components of bright yellow (Region-I), yellowish brown (Region-II), and dark brown (Region-III) were determined to be [0.820 0.715 $0.280]$, [ [ $\left.\begin{array}{lll}0.755 & 0.465 & 0.006\end{array}\right]$, and [ $\left.\begin{array}{llll}0.360 & 0.130 & 0.088\end{array}\right]$ in order to extract a feature that can be correlated with the level of acrylamide in potato chips. The image of interest was required to be segmented to obtain this feature. Pixels of fried potato images were classified into three sets based on their Euclidian distances to the above three representative mean values. Members of Set-I, Set-II, and Set-III are those pixels having the properties:

$$
\begin{aligned}
& \left\{\left(x_{r}(n, m)-0.820\right)^{2}+\left(x_{g}(n, m)-0.715\right)^{2}+\left(x_{b}(n, m)\right.\right. \\
& \left.\quad-0.280)^{2}\right\} \leq\left\{\left(x_{r}(n, m)-0.755\right)^{2}+\left(x_{g}(n, m)-0.465\right)^{2}\right. \\
& \left.+\left(x_{b}(n, m)-0.006\right)^{2}\right\}
\end{aligned}
$$

and

$$
\begin{aligned}
& \left.\left(x_{r}(n, m)-0.820\right)^{2}+\left(x_{g}(n, m)-0.715\right)^{2}+\left(x_{b}(n, m)-0.280\right)^{2}\right\} \\
& \quad \leq\left\{\left(x_{r}(n, m)-0.360\right)^{2}+\left(x_{g}(n, m)-0.130\right)^{2}+\left(x_{b}(n, m)\right.\right. \\
& \left.\quad-0.088)^{2}\right\} \\
& \left\{\left(x_{r}(n, m)-0.755\right)^{2}+\left(x_{g}(n, m)-0.465\right)^{2}\right. \\
& \left.\quad+\left(x_{b}(n, m)-0.006\right)^{2}\right\} \leq\left\{\left(x_{r}(n, m)-0.820\right)^{2}+\left(x_{g}(n, m)\right.\right. \\
& \left.\quad-0.715)^{2}+\left(x_{b}(n, m)-0.280\right)^{2}\right\}
\end{aligned}
$$

and

$$
\begin{aligned}
& \left\{\left(x_{r}(n, m)-0.755\right)^{2}+\left(x_{g}(n, m)-0.465\right)^{2}+\left(x_{b}(n, m)\right.\right. \\
& \left.-0.006)^{2}\right\} \leq\left\{\left(x_{r}(n, m)-0.360\right)^{2}+\left(x_{g}(n, m)-0.130\right)^{2}\right. \\
& \left.+\left(x_{b}(n, m)-0.088\right)^{2}\right\} \\
& \left\{\left(x_{r}(n, m)-0.360\right)^{2}+\left(x_{g}(n, m)-0.130\right)^{2}+\left(x_{b}(n, m)\right.\right. \\
& \left.-0.088)^{2}\right\} \leq\left\{\left(x_{r}(n, m)-0.820\right)^{2}+\left(x_{g}(n, m)-0.715\right)^{2}\right. \\
& \left.+\left(x_{b}(n, m)-0.280\right)^{2}\right\} \\
& \left\{\left(x_{r}(n, m)-0.360\right)^{2}+\left(x_{g}(n, m)-0.130\right)^{2}+\left(x_{b}(n, m)\right.\right. \\
& \left.-0.088)^{2}\right\} \leq\left\{\left(x_{r}(n, m)-0.755\right)^{2}+\left(x_{g}(n, m)-0.465\right)^{2}\right. \\
& \left.+\left(x_{b}(n, m)-0.006\right)^{2}\right\}
\end{aligned}
$$

respectively. 
Since yellowish brown colored potato chips were found to contain higher levels of acrylamide, a new parameter called the normalized area of Set-II pixels (NA2) was defined to correlate with the level of acrylamide. NA2 ratio was computed from the segmented images using the Matlab function called VectorQuantize implemented in this study. This function accepts two inputs: im_seq (image to be segmented) and $u$ (representative mean values). Representative cluster centers for Region-I, Region-II, and Region-III are held in vector $u$ together with an additional cluster center for background. This extra fourth value is to separate the background from the potato image. Typical mean values for the training images of potato chips were [0.95 0.950 .95$]$. In this specific case, vector u holds the values given below:

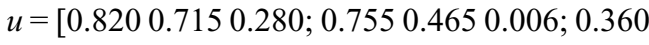

$$
\begin{aligned}
& 0.1300 .088 ; 0.950 .950 .95]^{\prime}
\end{aligned}
$$

Before the segmentation process, the Matlab function applies a symmetric median filter with [3 3] window to remove tiny oil sparks which may be present in the original potato chips images. The segmentation is carried on the filtered image held in im_seq by using a nearest mean classifier. Segmented image is displayed in a new window and the computed NA2 value is printed on the command windows at program termination using the following instruction:

seg_im = VectorQuantize(im_seq,u);

Figure 1 shows the original and segmented images of a potato chip sample using a semiautomatic segmentation algorithm described above. In this image, Set-II pixels occupy $19.96 \%$ of area of the entire image. Based on the results of image analyses performed on training samples, it was determined that there was a strong linear correlation between NA2 values and measured acrylamide levels of potato chips as shown in Fig. 2.

Acrylamide levels of number of commercial and laboratory-made fried potato chips $(n=60)$ were predicted by means of the correlation data obtained for training images. Measured and predicted acrylamide levels of potato chips are shown in Fig. 3. Percentage error of estimates was averaged as $29 \%$ with a SD of $21 \%$ for the test samples. From the viewpoint of food safety evaluation and inspection, it is important to define a threshold level for acrylamide in potato chips. However, no maximum permitted concentration has been established for acrylamide in thermally processed foods by the legal authorities. Once a critical level of acrylamide is adopted by the food industry, the semiautomatic segmentation algorithm may be used as a tool for safety evaluation and inspection purposes in order to sort out poor quality chips. We exemplified here the inspection capability of computer vision approach using the test samples. In this case, a provisional maximum permitted concentration of acrylamide was assumed to be $1000 \mathrm{ng} / \mathrm{g}$ in the

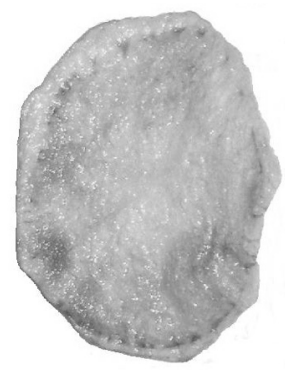

(a)

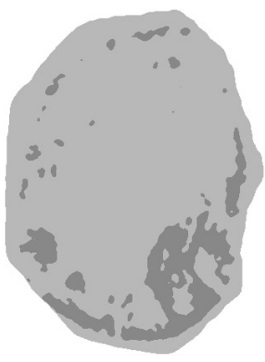

(b)
Figure 1. (a) Original fried potato chip image, (b) result of semiautomatic segmentation (NA2 $=0.1996)$.

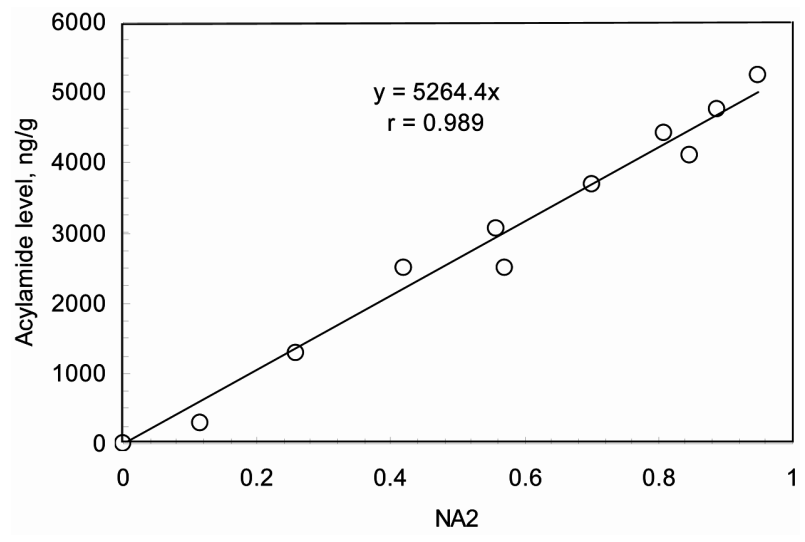

Figure 2. Correlation between defined parameter NA2 values and measured acrylamide levels for potato chips.

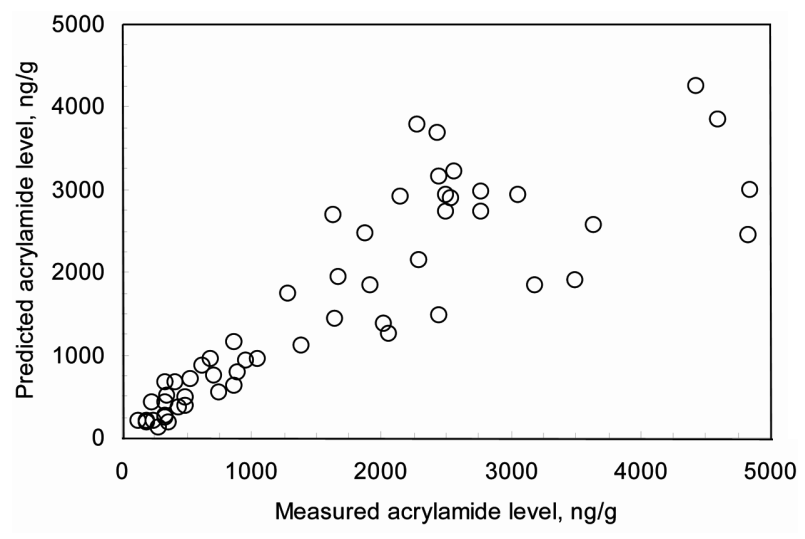

Figure 3. Measured and predicted acrylamide levels of test potato chips images.

finished product as a threshold limit. Based on the predicted acrylamide concentrations, potato chips exceeding the threshold limit were determined by image analysis. Potato chips exceeding the threshold limit could be accurately recognized by means of the image segmentation algorithm with one exception. One of the samples which had a measured acrylamide concentration of $1053 \mathrm{ng} / \mathrm{g}$ failed to be recognized since its predicted concentration was $948 \mathrm{ng} / \mathrm{g}$. 


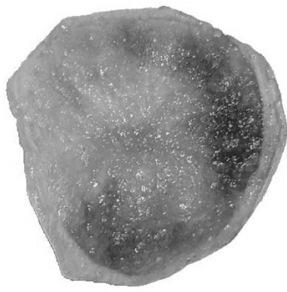

(a)

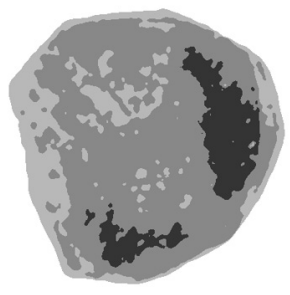

(b)
Figure 4. (a) Original and (b) segmented images of an overprocessed potato chip $(\mathrm{NA} 2=0.5946, \mathrm{NA} 3=0.1268)$.

The semiautomatic segmentation algorithm can also be used for the recognition of overprocessed potato chips. In this case, another parameter called normalized area of SetIII pixels (NA3) is defined. Pixel entries of Set-III indicate dark brown colored regions in the image of interest. Since the degree of overprocessing in potato chips is related to the NA3 ratio, a threshold limit may be defined to inspect over processed potato chips using the same algorithm. Figure 4 shows the original and segmented images of a potato chip sample. Since it was overprocessed, both yellowish brown and dark brown regions are clearly visible in this image in which Set-III pixels occupy $12.68 \%$ of the area of the entire image.

\section{Concluding remarks}

In the food industry, quality evaluation still heavily depends on manual inspection, which is tedious, laborious, and costly, and is easily influenced by physiological factors, inducing subjective and inconsistent evaluation results [25]. We described here a promising computer vision approach for the prediction of acrylamide level in potato chips. Featuring parameter NA2 was extracted from the digital images of potato chips and linearly correlated with the acrylamide level.

With the advantages of speed and accuracy, computer vision is considered to be a powerful tool for safety evaluation and inspection purposes for the food industry. In such a system, digital camera can be installed in the packaging line and fried potato images can be analyzed in real time and those products with NA2 values exceeding the predefined threshold limit. Present technology in terms of the speed of computers makes it possible to perform this kind of data processing in reasonable times. Inspection capability of the computer vision was confirmed for commercial and laboratory made potato chips assuming the provisional maximum permitted concentration of acrylamide in the finished product as $1000 \mathrm{ng} / \mathrm{g}$.
We thank Scientific and Technological Research Council of Turkey (Project no. TOVAG COST 927-2) and Turkish Academy of Sciences (TUBA-GEBIP Study Grant) for financial support, and Ankara Test and Analysis Laboratory for LC-MS analyses.

\section{References}

[1] Tareke, E., Rydberg, P., Karlsson, P., Törnqvist, M., Eriksson, S., Chem. Res. Toxicol. 2000, 13, 517-522.

[2] WHO, Report of a Joint FAO/WHO Consultation, 2002.

[3] Rosén, J., Hellenäs, K.-E., Analyst 2002, 127, 880-882.

[4] Ahn, J. S., Castle, L., Clarke, D. B., Lloyd, A. S., et al., Food Addit. Contam. 2002, 19, 1116-1124.

[5] Roach, J. A., Andrzejewski, D., Gay, M. L., Nortrup, D., Musser, S. M., J. Agric. Food Chem. 2003, 51, 7547-7554.

[6] Riediker, S., Stadler, R. H., J. Chromatogr. A 2003, 1020, $121-130$

[7] Andrzejewski, D., Roach, J. A. G., Gay, M. L., Musser, S. M., J. Agric. Food Chem. 2004, 52, 1996-2002.

[8] Hoenicke, K., Gatermann, R., Harder, W., Hartig, L., Anal. Chim. Acta 2004, 520, 207-216.

[9] Biedermann, M., Biedermann-Brem, S., Noti, A., Grob, K., et al., Mitt. Lebensm. Hyg. 2002, 93, 638-652.

[10] Pittet, A., Périsset, A., Oberson, J.-M., J. Chromatogr. A 2003, 1035, 123-130.

[11] Ballard, D. A., Brown, C. M., Computer Vision, PrenticeHall, Eaglewoood Cliffs, New Jersey 1982.

[12] Krutz, G. W., Gibson, H. G., Cassens, D. L., Zhang, M., Landwards 2000, 55, 2-9.

[13] Mottram, D. S., Wedzicha, B. L., Dodson, A. T., Nature 2002, $419,448-449$.

[14] Stadler, R. H., Blank, I., Varga, N., Robert, F. et al., Nature 2002, 419, 449-450.

[15] Friedman, M., J. Agric. Food Chem. 2003, 51, 4504-4526.

[16] Márquez, G., Anón, M. C., J. Food Sci. 1986, 51, 157-160.

[17] Amrein, T. M., Schönbächler, B., Escher, F., Amado, R., J. Agric. Food Chem. 2004, 52, 4282-4288.

[18] Surdyk, N., Rosén, J., Andersson, R., Åman, P., J. Agric. Food Chem. 2004, 52, 2047-2051.

[19] Pedrechi, F., Moyano, P., Kaack, K., Granby, K., Food Res. Intn'l. 2005, 38, 1-9.

[20] Senyuva, H. Z., Gökmen, V., Food Addit. Contam. 2005, 22, 214-220.

[21] Cetin, A. E., Weerackody, V., IEEE Trans. Circuits Syst. 1988, 35, 1550.

[22] Rabiner, L., Juang, B. H., Fundamentals of Speech Recognition, Prentice Hall, New Jersey 1993.

[23] Gökmen, V., Senyuva, H. Z., Acar, J., Sarioglu, K., J. Chromatogr. A 2005, 1088, 193-199.

[24] Gonzales, R. C., Woods, R. E., Digital Image Processing, Prentice-Hall, Eaglewood Cliffs, New Yersey 2002.

[25] Brosnan, T., Sun, D.-W., J. Food Eng. 2004, 61, 3-16. 


\section{Appendix}

\section{MatLab Code}

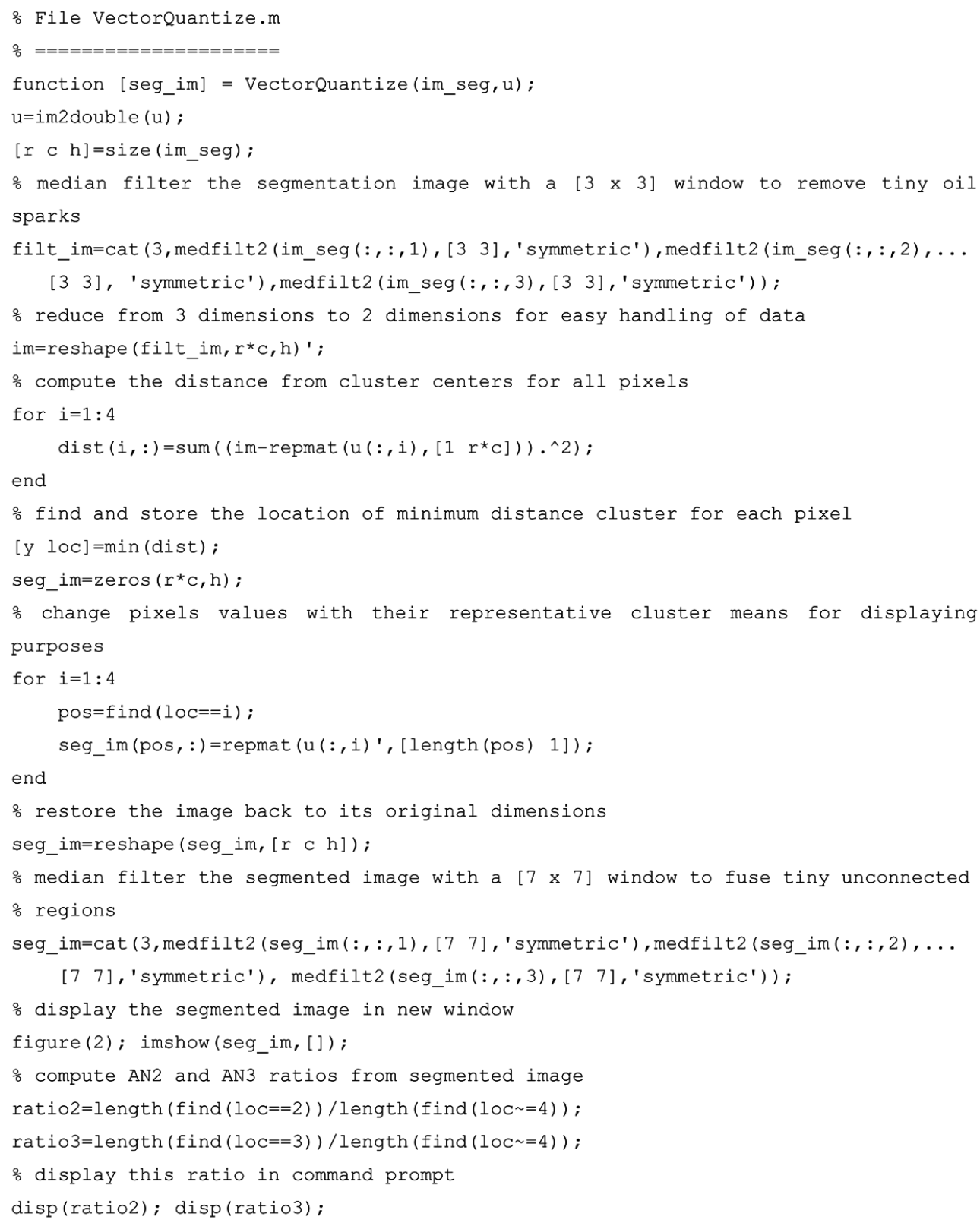

